\title{
CHARACTERIZATION OF NANOCRYSTALLINE SILICON GERMANIUM FILM AND NANOTUBE IN ADSORPTION GAS BY MONTE CARLO AND LANGEVIN DYNAMIC SIMULATION
}

\author{
M. Monajjemi ${ }^{1 *}$, L. Mahdavian ${ }^{1,2}$ and F. Mollaamin ${ }^{3}$ \\ ${ }^{1}$ Department of Chemistry, Islamic Azad University, Science and Research Branch, P. O. Box \\ 14155-775, Tehran, Iran \\ ${ }^{2}$ Department of Chemistry, Islamic Azad University, Doroud Branch, P. O. Box 133, Doroud, \\ Iran \\ ${ }^{3}$ Department of Chemistry, Qom Branch, Islamic Azad University, Qom, Iran
}

(Received July 17, 2007; revised November 28, 2007)

\begin{abstract}
The nanocrystalline silicon-germanium films (Si/Ge) and $\mathrm{Si} / \mathrm{Ge}$ nanotubes have low band gaps and high carrier mobility, thus offering appealing potential for absorbing gas molecules. Interaction between hydrogen molecules and bare as well as functionalized $\mathrm{Si} / \mathrm{Ge}$ nanofilm and nanotube was investigated using Monte Carlo (MC) and Langevin dynamic (LD) simulation methods. It was found that the binding energy of the $\mathrm{H}_{2}$ on the $\mathrm{Si} / \mathrm{Ge}$ surface is weak, and be enhanced by increasing curvature of surface to tube form and increasing temperature. The structural, total energy and energy band gaps of $\mathrm{H}_{2}$ absorbed nanocrystalline silicon germanium film $(\mathrm{Si} / \mathrm{Ge})$ and as it passes through $\mathrm{Si} / \mathrm{Ge}$ nanotube was also studied. They are computed with MC and LD simulation the methods at different temperatures. All the calculations were carried out using HyperChem 7.0 program package.
\end{abstract}

KEY WORDS: Monte Carlo, Langevin dynamic simulation, Silicon-germanium films (Si/Ge), Si/Ge nanotube

\section{INTRODUCTION}

$\mathrm{Si} / \mathrm{Ge}$ nanostructures are considered as candidates with high potential for integration into many nanoengineered systems. Moreover, their integration into micro-electric mechanical system (MEMS) opens new routes for the fabrication of ever faster and sensitive devices. Thus one key element is the development of methods to fabricate three-dimensional $\mathrm{Ge} / \mathrm{Si}$ micro- and nanoobjects (MNOs) and controls their size accurately [1]. Careful comparison of silicon and germanium chemical properties, however, shows subtle but indisputable differences, with Ge exhibiting distinct lithopile, siderophile or organophile behavior depending on its environment [2]. These specific properties are responsible for $\mathrm{Ge}$ geochemical cycles in the ocean and continental environments not being simple analogues of corresponding silica cycles [3]. This requires $\mathrm{Ge}$ to be enriched in the secondary phases that form during the weathering of primary silicates.

$\mathrm{Si} / \mathrm{Ge}$ is used for the adsorption of gases that is to form nanofilm and nanotube (Figure 1). It is important to study the e-beam induced phenomena in silica based glasses because germanium doped silica is one of the most used optical materials for production of integrated optical devices in which e-beam writing is foreseen. The integrated circuit fabrication by direct e-beam writing offers several advantages compared to the traditional photolithography techniques, in particular its large flexibility which allow the mastering of section of refractive index waveguides [4].

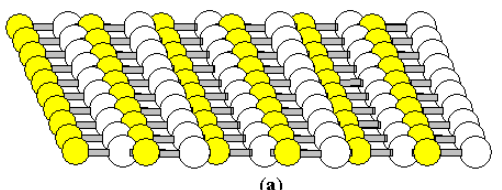

(a)

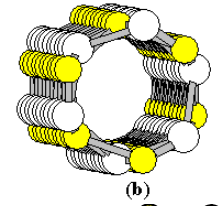

Figure 1. Optimized configuration top-view $\left(\mathrm{OSi}_{\mathrm{S}} \mathrm{OGe}\right)$ nanofilm and $(\mathrm{OSi} / \mathrm{OGe})$ nanotube.

*Corresponding author. E-mail: m_monajjemi@yahoo.com 
Following the observation of strong visible luminescence at room temperature from porous $\mathrm{Si}$ (PS) [5], several groups also attempted to prepare porous $\mathrm{Si} / \mathrm{Ge}$ (PSG) with visible luminescence, either by anodization or stain etching [6]. The second observation makes PSG a more attractive material than PS for both fundamental research and industrial applications.

However, further detailed investigations are required to determine the properties of such films and tubes that evaluate their potential for future application in technologies compatible with main stream $\mathrm{Si} / \mathrm{Ge}$ technology [1]. The sealed silica tube method is known as the most convenient and simplest ways for the preparation of sulfide compounds from the mixture of an element and sulfur. There is a lot of information available about the syntheses of sulfides by that method in the literature [7].

A classical example of a heterogeneous chemical reaction is recombinative desorption of two atoms from well-defined surfaces. In the elementary case the process involves a breaking of two adatom-surface bonds and formation of a diatomic molecule with a single new atom-atom bond [8]. However, such a trivial description of the process can neglect the role of a solid surface. Effects such as reconstruction of a surface, the local modification of electronic states or even the formation or breaking of chemical bonds between surface atoms can essentially influence energetic and dynamics of reactions and complicate the above mentioned elementary model. (While for metals the consideration of such effects is complicated, weakening or even breaking of bonds on a covalent solid surface is identified much easier.) In this connection a "hydrogen $\mathrm{Si} / \mathrm{Ge}$ ' system is an excellent example [9].

Hydrogen is an elementary adsorbate and at the same time it plays an important technological role. However, despite of intensive experimental and theoretical study of hydrogen interaction with a silicon surface, the details of mechanisms of $\mathrm{H}_{2}$ adsorption and desorption from a $\mathrm{Si} / \mathrm{Ge}$ surface remain contradictory. The basic contradiction is the first order of desorption kinetics and its recombinative character (hydrogen desorbs only in molecular form $\mathrm{H}_{2}$ ) [10] has required a detailed discussion of the desorption mechanism. From the recently proposed models it is necessary to note, for example, a desorption mediated by surface defects [11], concerted desorption from "pre-paired" configuration, the mechanism of irreversible excitation of hydrogen molecules in the delocalized state. Another contradiction is a very low sticking coefficient of molecular hydrogen, which assumes a high adsorption barrier, while in desorption experiments and theoretical calculations such which assumes a high adsorption barrier, while in desorption experiments and theoretical calculations such barrier was not observed. The influence of surface coverage on the character of interaction with hydrogen is not widely studied yet [12].

\section{EXPERIMENTAL}

\section{Computational details}

There are three steps in carrying out any quantum mechanical calculation in HyperChem 7.0 program package [13]. First, prepare a molecule with an appropriate starting geometry. Second, choose a calculation method and its associated (Setup menu) options. Third, choose the type of calculation (single point, geometry optimization, molecular dynamics, Langevin dynamics, Monte Carlo, or vibrational analysis) with the relevant (Compute menu) options. These geometry optimization calculations can use either molecular mechanics or quantum mechanics to further refine the molecular geometry beyond that given by the Model Builder.

HyperChem uses the Metropolis method [14], which chooses random configurations with this probability, to concentrate sampling of configurations in regions of space that make important contributions to the calculation of thermodynamic averages. 
Langevin dynamics $(L D)$ simulation

The Langevin equation is a stochastic differential equation in which two force terms have been added to Newton's second law to approximate the effects of neglected degrees of freedom [15]. These simulations can be much faster than molecular dynamics. The molecular dynamics method is useful for calculating the time dependent properties of an isolated molecule. However, more often, one is interested in the properties of a molecule that is interacting with other molecules. With HyperChem, we can add solvent molecules to the simulation explicitly, but the addition of many solvent molecules will make the simulation much slower. A faster solution is to simulate the motion of the molecule of interest using Langevin dynamics. Langevin dynamics simulates the effect of molecular collisions and the resulting dissipation of energy that occur in real solvents, without explicitly including solvent molecules. This is accomplished by adding a random force (to model the effect of collisions) and a frictional force (to model dissipative losses) to each atom at each time step. Mathematically, this is expressed by the Langevin equation of motion:

$$
\frac{\vec{F}_{i}}{m_{i}}-\gamma_{i} \vec{v}_{i}+\frac{\vec{R}_{i}(t)}{m_{i}}=\vec{a}_{i}
$$

Here, $F_{i}$ is force on each atom as a function of time, $\gamma_{i}$ is the friction coefficient of the solvent, in units of $\mathrm{ps}^{-1}$, and $R_{i}$ is the random force imparted to the solute atoms by the solvent and the change in velocities, $\hat{v}_{i}$, is equal to the integral of acceleration over time. The friction coefficient is related to the diffusion constant $\mathrm{D}$ of the solvent by Einstein's relation:

$\gamma_{i}=\frac{K_{B} T}{m D}$.

The random force is calculated as a random number, taken from a Gaussian distribution, with an average value of zero and no correlation with the atom's velocity.

\section{Integration algorithm}

To integrate the Langevin equation, HyperChem uses the method [16] of:

$$
\begin{aligned}
& r(t+\Delta t)=r(t)+c_{1} \Delta t v(t)+c_{2} \Delta t^{2} F(t) / m+\Delta r^{G} \\
& v(t+\Delta t)=c_{0} v(t)+c_{1} \Delta t F(t) / m+\Delta v^{G}
\end{aligned}
$$

where, if the atomic positions at a particular time, $t$, are $r(t)$, the velocities, $v(t)$ are the first derivative of the positions with respect to time

$$
\begin{aligned}
& \mathrm{c}_{0}=\mathrm{e}^{-\mathrm{t}} \\
& \mathrm{c}_{1}=\left(1-\mathrm{c}_{0}\right) /(\gamma \Delta \mathrm{t}) \\
& \mathrm{c}_{2}=\left(1-\mathrm{c}_{1}\right) /(\gamma \Delta \mathrm{t})
\end{aligned}
$$

That $\Delta r^{G}$ and $\Delta v^{G}$ are random numbers chosen from a Gaussian distribution, with:

$$
\begin{aligned}
& \left\langle\Delta r^{G}\right\rangle=0 \\
& \left\langle\Delta v^{G}\right\rangle=0
\end{aligned}
$$

And standard deviations:

$$
\sigma_{\mathrm{r}}^{2}=\Delta \mathrm{t}^{2}\left(\mathrm{k}_{\mathrm{B}} \mathrm{T} / \mathrm{m}\right)(\gamma \Delta \mathrm{t})^{-1}\left(2-(\gamma \Delta \mathrm{t})^{-1}\left(3-4 \mathrm{e}^{-\gamma \Delta \mathrm{t}}+\mathrm{e}^{-2 \gamma \Delta \mathrm{t}}\right)\right)
$$


$\sigma_{\mathrm{v}}^{2}=\left(\mathrm{k}_{\mathrm{B}} \mathrm{T} / \mathrm{m}\right)\left(1-\mathrm{e}^{-2 \gamma \Delta \mathrm{t}}\right)$

$\sigma_{\mathrm{r}}$ and $\sigma_{\mathrm{v}}$ are correlated, with correlation coefficient $c_{n}$, defined through the relation:

$c_{n} \sigma_{r} \sigma_{\mathrm{v}}=\Delta \mathrm{t}\left(\mathrm{k}_{\mathrm{B}} \mathrm{T} / \mathrm{m}\right)(\gamma \Delta \mathrm{t})^{-1}\left(1-\mathrm{e}^{-2 \gamma \Delta \mathrm{t}}\right)$

Assuming that the interatomic force term $\mathrm{F}(\mathrm{t})$ varies linearly with time, the equations above can be rewritten in a form which produces more accurate simulations:

$r(t+\Delta t)=r(t)+c_{1} \Delta t v(t)+c_{2} \Delta t^{2} F(t) / m+\Delta r^{G}$

$v(t+\Delta t)=c_{0} v(t)+\left(c_{1}-c_{2}\right) \Delta t F(t) / m+c_{2} \Delta t F(t+\Delta t) / m+\Delta v^{G}$

as $\gamma$ approaches zero, this method reduces to the velocity Verlet algorithm:

$r(t+\Delta t)=r(t)+\Delta t v(t)+1 / 2 \Delta t^{2} F(t) / m$

$\mathrm{v}(\mathrm{t}+\Delta \mathrm{t})=\mathrm{v}(\mathrm{t})+1 / 2 \Delta \mathrm{t}[\mathrm{F}(\mathrm{t})+\mathrm{F}(\mathrm{t}+\Delta \mathrm{t}) / \mathrm{m}]$

In general, Langevin dynamics simulations run much the same as molecular dynamics simulations. There are differences due to the presence of additional forces. Most of the earlier discussions on simulation parameters and strategies for molecular dynamics also apply to Langevin dynamics, exceptions and additional considerations are noted below.

\section{Molecular mechanics (Monte Carlo simulation)}

The Metropolis implementation of the Monte Carlo algorithm has been developed by studying the equilibrium thermodynamics of many-body systems. Choosing small trial moves, the trajectories obtained applying this algorithm agree with those obtained by Langevin's dynamics [17]. With effectively search in the resultant energy landscape, we exploited the recently developed parallel hyperbolic sampling algorithm in our Monte Carlo (MC) simulations. Previously, this protocol was shown to be more effective than general replica sampling in searching for low-energy structures. Especially the energy landscape is significantly more rugged for proteins of large size than the energy landscape of small proteins [18]. This is understandable because the Monte Carlo simulations always detect the so-called "important phase space" regions which are of low energy [19]. Because of imperfections of the force field, this lowest energy basin usually does not correspond to the native state in most cases, so the rank of native structure in those decoys produced by the force field itself is poor. Therefore, the rank of native structure could be relatively better when ranked by the second force field [20]. In order to compute the average properties from a microscopic description of a real system, one should evaluate integrals over phase space. It may be calculated for an $N$-particle system in an ensemble with distribution function $P\left(r^{N}\right)$, the experimental value of a property $A\left(r^{\mathrm{N}}\right)$ from:

$\left\langle A\left(r^{N}\right)\right\rangle=\int A\left(r^{N}\right) P\left(r^{N}\right) d r^{N}$

The problem with direct evaluation of this multi-dimensional integral (apart of the huge number of phase space points as a sample) is that most of the configurations sampled contribute nothing to the integral. Having energy is so high that the probability of their occurrence is vanishingly small. The trick, then, is to generate trajectories sample commonly occurring configurations more than rare ones.

For LD and MC calculation the energies are essentially the only quantities available from a single point calculation. An analysis of the components of this molecular mechanics energy is placed in the log file for further detail. In the case of $\mathrm{MM}^{+}$, a much more complete description of the individual contributions to the energy, including individual stretch energies, bending energies, etc., is placed in the log file along with the system dipole moment, if bond dipoles are available. 


\section{RESULTS AND DISCUSSION}

The interaction between adsorbed hydrogen molecular on surface $\mathrm{Si} / \mathrm{Ge}$ film and nanotube is investigated by using the first-principles calculation. $\mathrm{Si} / \mathrm{Ge}$ nanotube is a new form of $\mathrm{Si} / \mathrm{Ge}$ made by rolling up a single $\mathrm{Si} / \mathrm{Ge}$ sheet, so narrow tube opened at both ends.

It is found that the binding energy of the physisorbed $\mathrm{H}_{2}$ on $\mathrm{Si} / \mathrm{Ge}$ nanofilm surface is weak, and it can increase significantly in a nanotube. The character of the binding dramatically changes when passing molecular $\mathrm{H}_{2}$ functionalizes through it. Out of two adsorbed $\mathrm{H}_{2}$, the first one can be adsorbed dissociatively and the second one is chemisorbed molecularly. The nature of binding has a very weak physisorption in the third adsorbed $\mathrm{H}_{2}$. The $\mathrm{Si} / \mathrm{Ge}$, also, promotes the chemisorptions of $\mathrm{H}_{2}$ with smaller binding energy. The present results reveal the important effect of passing the molecular $\mathrm{H}_{2}$ through nanotube. These results will increase our understanding of the molecular and dissociative adsorption of hydrogen.

Simulations are run with the $\mathrm{H}_{2}$ bond length at $0.75 \AA$, which is the optimum bond length of free $\mathrm{H}_{2}$. Simulations are, also, run with the bond length at $1.16 \AA$, the optimized structure of $\mathrm{H}_{2}$ attached to $\mathrm{Si} / \mathrm{Ge}$ surface. The $\mathrm{H}-\mathrm{H}$ interaction when absorbed on the surface is weakened, and; therefore, the $\mathrm{H}-\mathrm{H}$ distance has increased to $1.16 \AA$.

The total binding energies, $\mathrm{E}_{\mathrm{T}}$, are obtained form the expression:

$E_{T}=E_{T}\left[H_{2}\right]+E_{T}[S i / G e]-E_{T}\left[H_{2}+S i / G e\right]$

The total energies of free or absorbed $\mathrm{H}_{2}$ on the $\mathrm{Si} / \mathrm{Ge}$ nanofilm and $\mathrm{Si} / \mathrm{Ge}$ nanotube, is at the same distance. The binding energies for both free $\mathrm{H}_{2},\left(\mathrm{E}_{\mathrm{T}}\left[\mathrm{H}_{2}\right]\right)$ and absorbed $\mathrm{H}_{2},\left(\mathrm{E}_{\mathrm{T}}\left[\mathrm{H}_{2}+\right.\right.$ $\mathrm{Si} / \mathrm{Ge}]$ ) are considered at the lowest ground state of the total energies (either magnetic or nonmagnetic); a bare $\mathrm{Si} / \mathrm{Ge}$ surface has a non-magnetic ground state with zero net spin. $\mathrm{E}_{\mathrm{T}}>0$, corresponds to optimized stable structure and indicates the bonding.

The molecular dynamics method is useful for calculating the time-dependent properties of an isolated molecule. However; more often, one of these properties of a molecule is interacting with other molecules. With HyperChem 7.0 program package [13], we can add $\mathrm{H}_{2}$ molecules into the simulation explicitly, but the addition of many molecules will make the simulation much slower. A faster solution is to simulate the motion of the molecule of interest using Langevin dynamics and Monte Carlo simulation. To integrate the Langevin equations $(2,3)$, HyperChem uses the method [16] in calculation.

Both simulations were at different temperatures; the time step and friction coefficient for the Langevin simulation were $10^{-6} \mathrm{ps}$ and $0.1 \mathrm{ps}^{-1}$, respectively [21, 22]. Monte Carlo simulations are commonly used to compute the average thermodynamic properties of a molecule or a system of molecules, and have been employed extensively in the study of the structure and equilibrium properties of molecules [14]. Monte Carlo calculations are similar to the molecular (or Langevin) dynamics calculations (discussed previously). Monte Carlo simulations employ a statistical sampling technique to generate configurations, which represent a trajectory in phase space. Thus, unlike molecular dynamics or Langevin dynamics, which calculate ensemble averages by calculating averages over time, Monte Carlo calculations evaluate the averages of the ensemble directly by sampling configurations from the statistical ensemble. If the run takes enough time, Monte Carlo and molecular dynamics must give the same average results for the same system. However, only molecular dynamics can calculate time-dependent properties of a system, such as rotational frequencies or transitional rates. On the other hand, Monte Carlo is generally better in sampling the allowed states of a system, and; thus, can often calculate the average properties more quickly and accurately (Figures 2,3) when the temperature is increased the total energy is increased too. Therefore, the bonding distance is $1.16 \AA$, the total energy began to increase in low temperatures, which the total energy is stable in LD method and irregular in MC method when the temperature is high. 


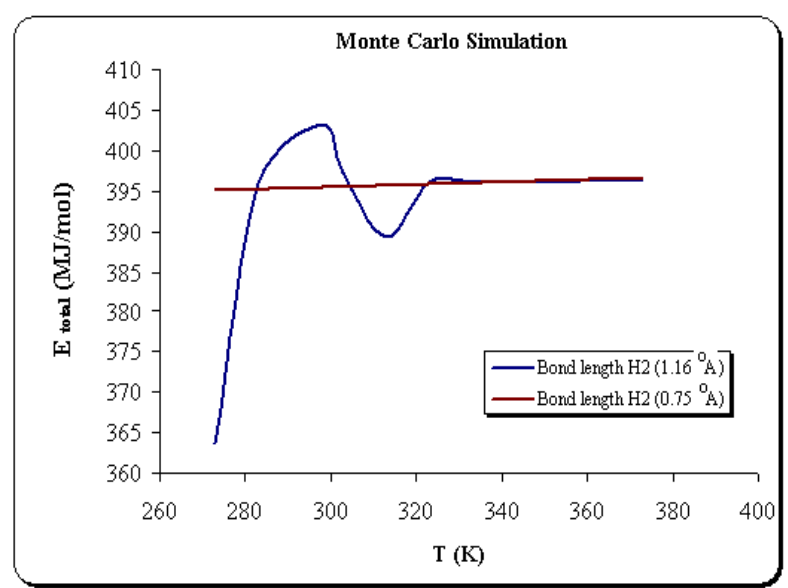

Figure 2. The total energy (MJ/mol) calculated for adsorption $\mathrm{H}_{2}$ with different bond lengths on surface of Si-Ge film by Monte Carlo Simulation.

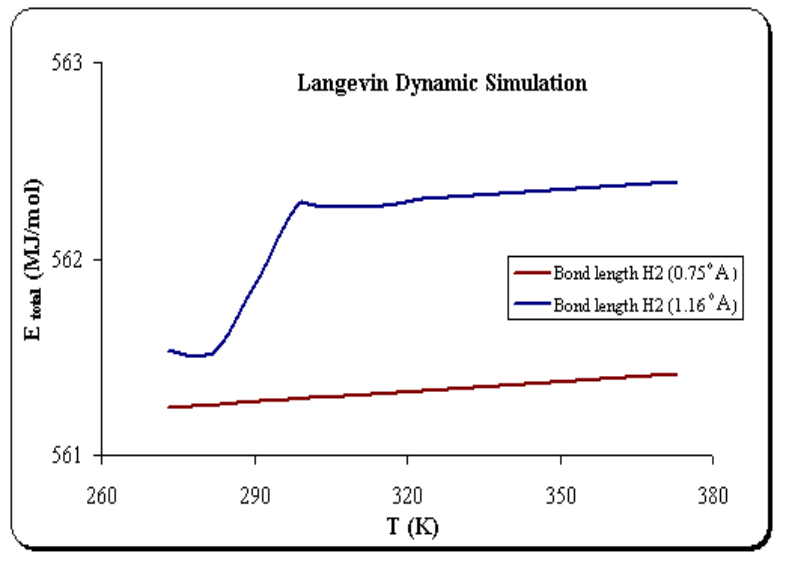

Figure 3. The total energy $(\mathrm{MJ} / \mathrm{mol})$ calculated for adsorption $\mathrm{H}_{2}$ with different bond lengths on surface of Si-Ge film by Langevin dynamic simulation.

Table 1. The total energy, potential energy and kinetic energy $(\mathrm{MJ} / \mathrm{mol})$ calculated for adsorption of $\mathrm{H}_{2}$ with different bond length on surface of $\mathrm{Si} / \mathrm{Ge}$ film by Monte Carlo and Langevin daynamic simulation.

\begin{tabular}{|c|c|c|c|c|c|c|c|c|c|c|c|c|}
\hline \multirow{3}{*}{$\mathrm{T}(\mathrm{K})$} & \multicolumn{6}{|c|}{ Monte Carlo } & \multicolumn{6}{|c|}{ Langevin dynamic } \\
\hline & \multicolumn{3}{|c|}{ Bond length $\mathrm{H}_{2}(0.75 \AA)$} & \multicolumn{3}{|c|}{ Bond length $\mathrm{H}_{2}(1.16 \AA)$} & \multicolumn{3}{|c|}{ Bond length $\mathrm{H}_{2}(0.75 \AA)$} & \multicolumn{3}{|c|}{ Bond length $\mathrm{H}_{2}(1.16 \AA)$} \\
\hline & $\mathrm{E}_{\text {Total }}$ & $\mathrm{E}_{\text {Pot }}$ & $\mathrm{E}_{\mathrm{Kin}}$ & $\mathrm{E}_{\text {Total }}$ & $\mathrm{E}_{\text {Pot }}$ & $\mathrm{E}_{\mathrm{Kin}}$ & $\mathrm{E}_{\text {Total }}$ & $\mathrm{E}_{\text {Pot }}$ & $\mathrm{E}_{\mathrm{Kin}}$ & $\mathrm{E}_{\text {Total }}$ & $\mathrm{E}_{\text {Pot }}$ & $\mathrm{E}_{\mathrm{Kin}}$ \\
\hline 273 & 392.80 & 392.31 & 0.49 & 363.61 & 363.14 & 0.48 & 561.24 & 328.77 & 232.47 & 561.53 & 329.20 & 232.33 \\
\hline 298 & 395.93 & 395.40 & 0.53 & 403.15 & 402.63 & 0.52 & 561.28 & 328.75 & 232.53 & 56 & 329.07 & 233.20 \\
\hline 313 & 396.40 & 395.84 & 0.56 & \begin{tabular}{|l|}
389.37 \\
\end{tabular} & 388.82 & 0.55 & 561.31 & 328.74 & 232.57 & \begin{tabular}{|l|}
562.27 \\
\end{tabular} & \begin{tabular}{|l|}
329.07 \\
\end{tabular} & 233.20 \\
\hline 323 & 7.12 & 396.55 & 0.57 & 396.08 & 395.51 & 0.57 & 561.33 & 328.74 & 232.59 & 562.31 & 329.07 & 233.24 \\
\hline 333 & 397.24 & 396.65 & 0.59 & 396.06 & 395.48 & 0.58 & 561.34 & 328.73 & 232.61 & 562.33 & 329.07 & 233.26 \\
\hline 343 & 397.75 & 397.14 & 0.61 & 396.04 & 395.44 & 0.60 & 561.36 & 328.73 & 232.64 & 562.35 & 329.06 & 233.28 \\
\hline 353 & 395.68 & 395.06 & 0.63 & \begin{tabular}{|l|}
396.17 \\
\end{tabular} & 395.55 & 0.62 & 561.38 & 328.72 & 232.66 & 562.36 & 329.06 & 233.30 \\
\hline 363 & 395.64 & 395.00 & 0.65 & 396.35 & 395.71 & 0.64 & 561.40 & 328.72 & 232.68 & 562.38 & 329.06 & 233.32 \\
\hline
\end{tabular}

Bull. Chem. Soc. Ethiop. 2008, 22(2) 
The results show which the interaction between $\mathrm{H}_{2}$ and surface of $\mathrm{Si} / \mathrm{Ge}$ nanofilm is endothermic. In Table 1, the total energy, potential energy and kinetic energy are calculated by Monte Carlo and Langevin dynamic simulation. Therefore, we tried to investigate the continued of converting surface $\mathrm{Si} / \mathrm{Ge}$ nanofilm to nanotube. The molecular $\mathrm{H}_{2}$ with bond lengths $(0.75$ and $1.16 \AA$ ) is passing through $\mathrm{Si} / \mathrm{Ge}$ nanotube at various temperatures. The calculations are done by both methods (Tables 2 and 3). The results of both surfaces show which the binding energy for molecular $\mathrm{H}_{2}$ interaction in $\mathrm{Si} / \mathrm{Ge}$ nanotube is more increasing than in $\mathrm{Si} / \mathrm{Ge}$ nanofilm. In nanotube, various charge locations are created in nanotube when the surface turned into nanotube. These locations are observed at $353 \mathrm{~K}$ in Figures 4 and 5.

Table.2. The total energy and kinetic energy $(\mathrm{MJ} / \mathrm{mol})$ calculated for across $\mathrm{H}_{2}$ with bond lengths in $\mathrm{Si} / \mathrm{Ge}$ nanotube by Monte Carlo simulation.

\begin{tabular}{|c|c|c|c|c|c|c|c|c|c|c|c|c|c|c|c|c|}
\hline \multirow{3}{*}{$\begin{array}{c}\text { Distance } \\
\AA\end{array}$} & \multicolumn{16}{|c|}{ Bond length $\mathrm{H}_{2}(0.75 \AA)$} \\
\hline & \multicolumn{2}{|c|}{$273 \mathrm{~K}$} & \multicolumn{2}{|c|}{$298 \mathrm{~K}$} & \multicolumn{2}{|c|}{$313 \mathrm{~K}$} & \multicolumn{2}{|c|}{$323 \mathrm{~K}$} & \multicolumn{2}{|c|}{$333 \mathrm{~K}$} & \multicolumn{2}{|c|}{$343 \mathrm{~K}$} & \multicolumn{2}{|c|}{$353 \mathrm{~K}$} & \multicolumn{2}{|c|}{$363 \mathrm{~K}$} \\
\hline & E total & $\begin{array}{c}\mathrm{E} \\
\text { kin }\end{array}$ & E total & $\begin{array}{c}\mathrm{E} \\
\text { kin }\end{array}$ & E total & $\begin{array}{c}E \\
\text { kin }\end{array}$ & E total & $\begin{array}{c}\mathrm{E} \\
\text { kin }\end{array}$ & E total & $\begin{array}{c}\mathrm{E} \\
\text { kin }\end{array}$ & E total & \begin{tabular}{|c|}
$\mathrm{E}$ \\
kin
\end{tabular} & E total & \begin{tabular}{|c|} 
\\
kin
\end{tabular} & E total & $\begin{array}{c}\mathrm{E} \\
\text { kin }\end{array}$ \\
\hline 0 & 392.45 & 0.42 & 613.49 & 0.46 & 613.57 & 0.48 & 613.62 & 0.49 & 613.86 & 0.51 & 613.48 & 0.52 & 575.85 & 0.54 & 613.72 & 0.55 \\
\hline 5.77 & 614.20 & 0.42 & 615.01 & 0.46 & 614.39 & 0.48 & 614.54 & 0.49 & 613.86 & 0.51 & 614.45 & 0.52 & 614.54 & 0.54 & 614.39 & 0.55 \\
\hline 10.01 & 614.04 & 0.42 & 614.17 & 0.46 & 614.19 & 0.48 & 614.47 & 0.49 & 614.46 & 0.51 & 614.07 & 0.52 & 614.58 & 0.54 & 614.59 & 0.55 \\
\hline 14.93 & 614.96 & 0.42 & 614.97 & 0.46 & 614.31 & 0.48 & 614.50 & 0.49 & 614.42 & 0.51 & 614.49 & 0.52 & 614.64 & 0.54 & 614.65 & 0.55 \\
\hline 19.82 & 614.19 & 0.42 & 615.13 & 0.46 & 614.24 & 0.48 & 614.34 & 0.49 & 614.42 & 0.51 & 576.46 & 0.58 & 614.69 & 0.54 & 614.77 & 0.55 \\
\hline 23.41 & 614.43 & 0.42 & 614.86 & 0.46 & 615.09 & 0.48 & 615.09 & 0.49 & 615.53 & 0.51 & 614.50 & 0.52 & 615.50 & 0.54 & 615.56 & 0.55 \\
\hline \multirow{3}{*}{$\begin{array}{c}\text { Distance } \\
\AA\end{array}$} & \multicolumn{16}{|c|}{ Bond length $\mathrm{H}_{2}(1.16 \AA)$} \\
\hline & \multicolumn{2}{|c|}{$273 \mathrm{~K}$} & \multicolumn{2}{|c|}{$298 \mathrm{~K}$} & \multicolumn{2}{|c|}{$313 \mathrm{~K}$} & \multicolumn{2}{|c|}{$323 \mathrm{~K}$} & \multicolumn{2}{|c|}{$333 \mathrm{~K}$} & \multicolumn{2}{|c|}{$343 \mathrm{~K}$} & \multicolumn{2}{|c|}{$353 \mathrm{~K}$} & \multicolumn{2}{|c|}{$363 \mathrm{~K}$} \\
\hline & E total & $\begin{array}{c}\mathrm{E} \\
\text { kin } \\
\end{array}$ & E total & \begin{tabular}{|c}
$\mathrm{E}$ \\
kin
\end{tabular} & E total & $\begin{array}{c}\mathrm{E} \\
\text { kin }\end{array}$ & E total & $\begin{array}{c}\mathrm{E} \\
\text { kin }\end{array}$ & E total & $\begin{array}{c}\mathrm{E} \\
\text { kin } \\
\end{array}$ & E total & \begin{tabular}{|c}
$\mathrm{E}$ \\
kin
\end{tabular} & E total & \begin{tabular}{|c|}
$\mathrm{E}$ \\
kin
\end{tabular} & E total & $\begin{array}{c}\mathrm{E} \\
\text { kin }\end{array}$ \\
\hline 0 & 575.46 & 0.42 & 615.32 & 0.46 & 615.11 & 0.48 & 615.10 & 0.49 & 615.36 & 0.51 & 615.30 & 0.52 & 615.41 & 0.54 & 615.52 & 0.55 \\
\hline 5.77 & 614.50 & 0.42 & 614.37 & 0.46 & 613.93 & 0.48 & 614.31 & 0.49 & 614.36 & 0.51 & 614.27 & 0.52 & 614.42 & 0.54 & 614.79 & 0.55 \\
\hline 10.01 & 614.92 & 0.42 & 615.27 & 0.46 & 614.78 & 0.48 & 615.47 & 0.49 & 575.37 & 0.51 & 615.42 & 0.52 & 615.51 & 0.54 & 615.55 & 0.55 \\
\hline 14.93 & 614.86 & 0.42 & 615.08 & 0.46 & 613.99 & 0.48 & 614.29 & 0.49 & 614.16 & 0.51 & 614.36 & 0.52 & 615.07 & 0.54 & 614.99 & 0.55 \\
\hline 19.82 & 615.27 & 0.42 & 615.32 & 0.46 & 615.35 & 0.48 & 614.97 & 0.49 & 615.38 & 0.51 & 614.72 & 0.54 & 614.72 & 0.54 & 614.67 & 0.55 \\
\hline 23.41 & 614.68 & 0.42 & 614.87 & 0.46 & 615.25 & 0.48 & 614.21 & 0.49 & 614.29 & 0.51 & 614.84 & 0.54 & 614.84 & 0.54 & 614.61 & 0.55 \\
\hline
\end{tabular}

Nanostructures have considerably higher surface areas than their bulk counterparts; therefore, the surfaces often play important, sometimes even dominant, roles in the nanostructure properties. Figures 4 and 5 show the variation of $\mathrm{E}_{\mathrm{T}}$ for passing $\mathrm{H}_{2}$ through $\mathrm{Si} / \mathrm{Ge}$ nanotube at $353 \mathrm{~K}$. For hydrogen with bond length of $0.75 \AA$, at first, the total energy increases when passing $\mathrm{H}_{2}$ in the initial length of the nanotube, then it is stable to the end in Monte Carlo simulation. This process is not stable for hydrogen with $1.16 \AA$ length by this method. Then in the middle of length the nanotube, it begins to increase again and at the end of it begins to decrease. The minimum value of the interaction $\mathrm{E}_{\mathrm{T}}$ is $614.27 \mathrm{MJ} / \mathrm{mol}$. In Langevin dynamic simulation method, the total energy begins to decrease in the initial length of the nanotube for $\mathrm{H}_{2}$ with $0.75 \AA$ bond length.

But the passing $\mathrm{H}_{2}$ with $1.16 \AA$ bond length, in this method, increases in the initial length, and; then, it decreases at the end length of nanotube Figure 5. The total energy and kinetic energy $(\mathrm{MJ} / \mathrm{mol})$ are calculated for passing $\mathrm{H}_{2}$ with bond length $0.75 \AA$ and $1.16 \AA$ through $\mathrm{Si} / \mathrm{Ge}$ nanotube by both methods Tables 2 and 3 . 
Table.3. The total energy and kinetic energy $(\mathrm{MJ} / \mathrm{mol})$ calculated for across $\mathrm{H}_{2}$ with bond lengths in $\mathrm{Si} / \mathrm{Ge}$ nanotube by Langevin dynamic simulation.

\begin{tabular}{|c|c|c|c|c|c|c|c|c|c|c|c|c|c|}
\hline \multirow{3}{*}{$\begin{array}{c}\text { Distance } \\
\AA\end{array}$} & \multicolumn{13}{|c|}{ Bond length $\mathrm{H}_{2}(0.75 \AA)$} \\
\hline & \multicolumn{2}{|c|}{$273 \mathrm{~K}$} & \multicolumn{2}{|c|}{$298 \mathrm{~K}$} & $313 \mathrm{~K}$ & \multicolumn{2}{|c|}{$323 \mathrm{~K}$} & \multicolumn{2}{|c|}{$333 \mathrm{~K}$} & $343 \mathrm{~K}$ & $353 \mathrm{~K}$ & \multicolumn{2}{|c|}{$363 \mathrm{~K}$} \\
\hline & E total & E kin & E total & E kin & E total E kin & E total & E kin & E total & E kin & E total E kin & E total E kin & E total & E kin \\
\hline 0 & 656.64 & 71.24 & 656.65 & 571.26 & 657.3671 .38 & 657.37 & 71.40 & 657.39 & 71.42 & 657.4071 .44 & 657.4271 .45 & 657.43 & 71.47 \\
\hline 5.77 & 644.01 & 68.31 & 656.77 & 771.44 & \begin{tabular}{|l|l|}
657.47 & 71.56 \\
\end{tabular} & 657.49 & 71.58 & 396.90 & 16.18 & \begin{tabular}{|l|l|}
396.88 & 16.20 \\
\end{tabular} & 396.8916 .22 & 396.91 & 16.24 \\
\hline 10.01 & 656.75 & 71.41 & 656.77 & 71.43 & 657.4771 .55 & 657.49 & 71.57 & 657.50 & 71.59 & 657.5271 .60 & 657.5371 .62 & 657.55 & 51.64 \\
\hline 14.93 & 656.75 & 71.42 & 656.77 & 71.43 & \begin{tabular}{|l|l|}
657.47 & 71.56 \\
\end{tabular} & 657.49 & 71.58 & 657.50 & 71.59 & \begin{tabular}{|l|l|}
657.52 & 71.61 \\
\end{tabular} & \begin{tabular}{|l|l|}
657.53 & 71.63 \\
\end{tabular} & 657.55 & 71.65 \\
\hline 19.82 & 656.76 & 71.42 & 656.77 & 71.44 & \begin{tabular}{|l|l|}
657.47 & 71.56 \\
\end{tabular} & 657.49 & 71.58 & 657.50 & 71.60 & 657.527 & \begin{tabular}{|l|l|}
657.53 & 71.63 \\
\end{tabular} & 657.55 & 51.65 \\
\hline 23.41 & 656.72 & 71.35 & 656.74 & 71.37 & 657.4471 .50 & 657.46 & 71.51 & 657.47 & 71.53 & \begin{tabular}{|l|l|}
657.49 & 71.55 \\
\end{tabular} & 657.5071 .57 & 657.52 & 71.58 \\
\hline \multirow{3}{*}{$\begin{array}{c}\text { Distance } \\
\AA\end{array}$} & \multicolumn{13}{|c|}{ Bond length $\mathrm{H}_{2}(1.16 \AA)$} \\
\hline & \multicolumn{2}{|c|}{$273 \mathrm{~K}$} & \multicolumn{2}{|c|}{$298 \mathrm{~K}$} & $313 \mathrm{~K}$ & \multicolumn{2}{|c|}{$323 \mathrm{~K}$} & \multicolumn{2}{|c|}{$333 \mathrm{~K}$} & $343 \mathrm{~K}$ & $353 \mathrm{~K}$ & \multicolumn{2}{|c|}{$363 \mathrm{~K}$} \\
\hline & E total & E kin & E total & E kin & E total E kin & E total & E kin & E total & E kin & E total E kin & E total $\mathrm{E}$ kin & E total & E kin \\
\hline 0 & 657.84 & 71.68 & 657.69 & 71.47 & 657.7171 .50 & 657.73 & 71.52 & 657.74 & 71.53 & 657.7671 .55 & 657.7771 .57 & 657.79 & 71.59 \\
\hline 5.77 & 657.94 & 71.88 & 657.79 & 71.67 & \begin{tabular}{|l|l|l|}
657.81 & 71.69 \\
\end{tabular} & 657.83 & 71.71 & 657.74 & 71.53 & 657.8671 .75 & 657.8771 .76 & 657.89 & 71.78 \\
\hline 10.01 & 657.75 & 71.57 & 657.79 & 71.62 & 657.8171 .64 & 657.83 & 71.66 & 657.84 & 71.68 & 657.8671 .70 & \begin{tabular}{|l|l|}
657.87 & 71.71 \\
\end{tabular} & 657.89 & 71.73 \\
\hline 14.93 & 657.75 & 71.61 & 657.79 & 71.66 & $657.81 \quad 71.69$ & 657.83 & 71.70 & 657.84 & 71.72 & 657.8671 .74 & \begin{tabular}{|l|l|}
657.87 & 71.76 \\
\end{tabular} & 657.89 & 71.78 \\
\hline 19.82 & 657.75 & 71.58 & 657.79 & 71.63 & 657.8271 .65 & 657.83 & 71.67 & 657.85 & 71.69 & 657.8671 .70 & 657.8871 .72 & 657.89 & 71.74 \\
\hline 23.41 & 657.67 & 71.49 & 657.71 & $7=1$ & \begin{tabular}{|l|l|}
657.73 & 71.57 \\
\end{tabular} & 657.75 & $71=0$ & 65776 & 7160 & 657.7871 .62 & 657797164 & 65781 & 7166 \\
\hline
\end{tabular}

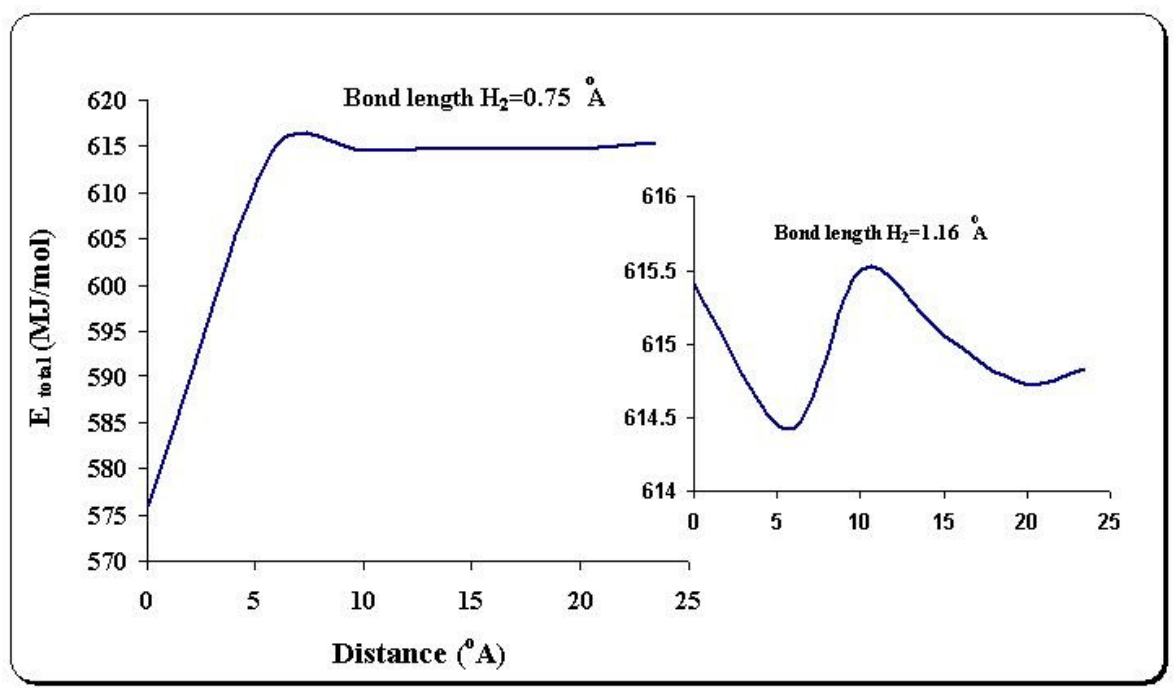

Figure 4. The total energy $(\mathrm{MJ} / \mathrm{mol})$ calculated for across $\mathrm{H}_{2}$ with bond lengths in $\mathrm{Si} / \mathrm{Ge}$ nanotube by Monte Carlo Simulation at $353 \mathrm{~K}$. 


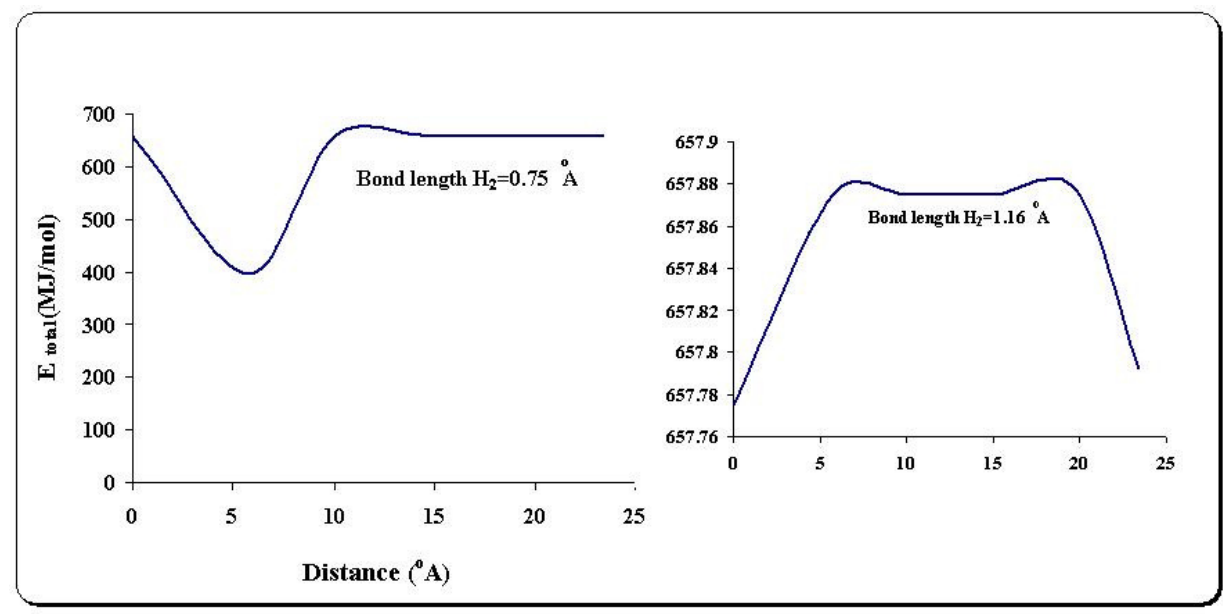

Figure 5. The total energy total $(\mathrm{MJ} / \mathrm{mol})$ calculated for across $\mathrm{H}_{2}$ with bond lengths in $\mathrm{Si} / \mathrm{Ge}$ nanotube by Langevin dynamic simulation at $353 \mathrm{~K}$.

\section{CONCLUSION}

In this work, we present a detailed analysis of the interaction between hydrogen molecules on the surface of $\mathrm{Si} / \mathrm{Ge}$ nanofilm and by passing $\mathrm{H}_{2}$ through $\mathrm{Si} / \mathrm{Ge}$ nanotube at different temperatures. These calculations are done by Monte Carlo and Langevin dynamic simulation in HyperChem 7.0 package program. In general, Langevin dynamics simulations are the same as molecular dynamics simulations. There are differences due to the presence of additional forces. Most of the earlier discussions on simulation parameters and strategies for molecular dynamics had been applied to Langevin dynamics.

We found that the binding energy between $\mathrm{H}_{2}$ molecule and outer surface of a bare $\mathrm{Si} / \mathrm{Ge}$ is very weak and the physisorption bond can easily be broken. The H-H interaction when absorbed on the surface is weakened, and; therefore, the $\mathrm{H}-\mathrm{H}$ distance has increased to $1.16 \AA$. The results have shown that the bond in the molecular interaction of $\mathrm{H}_{2}$ is relatively stronger with a shorter bond length, so energy changes are regular when passing $\mathrm{H}_{2}$ through nanotube. The binding energy would increase if $\mathrm{H}_{2}$ molecule was directly attached to $\mathrm{Si} / \mathrm{Ge}$ surface. However, the case with the $\mathrm{Si} / \mathrm{Ge}$ atom leads to a relative weaker interaction and smaller binding energies. $\mathrm{MC}$ and LD simulation were run for the interaction $\mathrm{H}_{2}$ and the surface results in a kind of binding between chemisorptions and physisorptions the binding energy. In this case, the $\mathrm{H}-\mathrm{H}$ bond length is stretched a bit from the normal value 0.75 to $1.16 \AA$. When the temperature is increased, the total energy, kinetic and potential energy are enhanced, so this interaction is endothermic. The calculated energy for the two systems shows that nanotube has considerably higher surface areas than their most counterparts.

\section{REFERENCES}

1. Zhang, L.; Golod, S.V.; Deckardt, E.; Prinz, V.; Gr.utzmacher, D. Physica. E. 2004, 23, 280.

2. Bernstein, L.R. Geochim. Cosmochim. Acta 1985, 49, 2409.

3. Pokrovsky, O.S.; Pokrovski, G.S.; Schott, J.; Galy, A. Geochim. Cosmochim. Acta 2006, 70, 3325 . 
4. Jacqueline, A.S.; Poumellec, B. J. Non-Crystalline Solids 2005, 351, 1196.

5. Canham, L.T. Appl. Phys. Lett. 1990, 57, 1046.

6. Kartopu, G.; Ekinci, Y.; Thin Solid Films 2005, 473, 213.

7. Wada, H.; Takada, K.; Sasaki, T. Solid State Ionics 2004, 172, 421.

8. Yanovsky, A.S.; Kolomoets, S.V. Vacuum 1999, 54, 47.

9. Stoker, M.W.; Merchant, T.; Morton, A.; Hildreth, J. Microelectronic Engineering 2003, 69 633.

10. Gupta, P.; Colvin, VL.; George, S. M. Phys. Rev. B 1988, 37, 8234.

11. Gross, A.; Bockstedte, M.; Scheßer, M. Phys. Rev. Lett. 1995, 75, 2718.

12. Colonna, S.; Terrasi, A.; Scalese, S.; Iacona, F.; Raineri,V.; La Via c, f.; Mobilio, S. Surface Science 2003, 532-535, 746.

13. HyperChem 7.0, Hypecube Inc., Gainesville, FL, USA, 2001.

14. Metropolis, N.; Rosenbluth, A.W.; Rosenbluth, M.N.; Teller, A.H.; Teller, E. J. Chem. Phys. 1953, 21, 1087.

15. Wang, W.; Skeel, R.D. Mol. Phys. 2003, 101, 2149.

16. Allen, M.P.; Tildesley, D.J. Computer Simulation of Liquids, Clarendon Press: Oxford, 1987, Ch.9, p. 261.

17. Tiana, G.; Sutto, L.; Broglia, R.A.; Physica A: Statistical Mechanics and its Applications 2007, 380, 241.

18. Strecker, N.; Moroz, V.; Jaraiz, M. Proceedings of the 2002 International Conference on Computational Nanoscience, 2002, p 247.

19. Liu, J.Ch.; Monson, P.A. Adsorption 2005, 11, 5.

20. Delville, A. J. Phys. Chem.B 2004, 108, 9984.

21. van Gunsteren, W.F.; Berendsen, H.J.C.; Rullmann, J.A.C. Molecular Physics 1981, 44, 69.

22. Chan, S.P.; Chen, G.; Gong, X.G.; Liu, Z.F. Phys. Rev. Lett. 2001, 87, 205. 